\title{
Les initiatives locales et les défis des acteurs du milieu face aux coupes et aux changements actuels
}

\author{
Marco Alberio \\ Université du Québec à Rimouski
}

\section{INTRODUCTION}

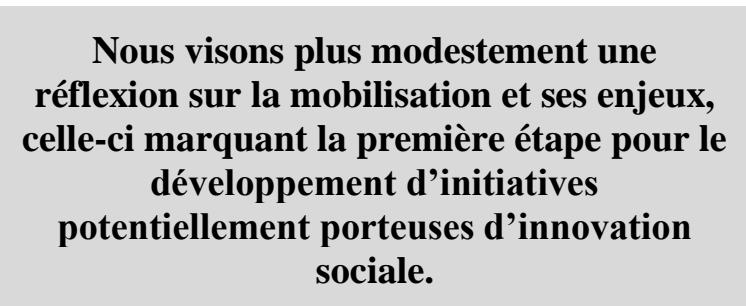

Dans cet article, nous présenterons des pistes de réflexion autour de la question de l'austérité et des transformations institutionnelles en cours, qui risquent à notre avis d'affecter à long terme le modèle de développement social et territorial québécois, traditionnellement basé sur la participation et la mobilisation citoyennes, de même que sur sa capacité d'inclusion. Nous tenons à souligner que cette réflexion est encore à une étape préliminaire, étant donné que le projet de loi 28 , regroupant une série de mesures ${ }^{1}$ visant à atteindre l'équilibre budgétaire, a été approuvé depuis seulement quelques mois. Les effets réels de ces mesures restent donc difficiles à identifier et surtout à mesurer.

Notre objectif n'est donc pas de démontrer avec une reddition de compte détaillée la corrélation entre contraintes budgétaires, réduction des financements aux initiatives locales et mise à risque de la concertation. De plus, comme on l'observera, les mesures d'austérité ne concernent pas que les aspects financiers, mais, entre autres, l'appréciation/évaluation des activités. Dans cet article, nous visons plus modestement une réflexion sur la mobilisation et ses enjeux, celle- ci marquant la première étape pour le développement d'initiatives potentiellement porteuses d'innovation sociale.

D'un point de vue méthodologique, nos arguments seront appuyés par des entretiens utilisés comme témoignages, afin de clarifier les caractéristiques intrinsèques de la participation et ses besoins, et de mieux comprendre pourquoi celle-ci, de par sa nature même, risque d'être mise en danger par l'austérité ${ }^{2}$. De plus, ces entretiens nous permettront de nous interroger sur la perception que certains intervenants ont des mesures d'austérité et sur la manière dont les communautés comptent répondre aux nouveaux impératifs. Ces perceptions ne mesurent naturellement pas l'effet de l'austérité, mais nous permettront, dans une perspective qualitative, d'éclaircir les motivations des acteurs concernés et de mieux comprendre quelques-unes de leurs difficultés.

Selon notre propos, certaines décisions d'ordre politique et institutionnel - réelles ou encore annoncées au moment des entrevues -, telles que les coupes budgétaires, la réorganisation des services sociaux et de santé, la fermeture ou, dans quelques cas, la réorganisation des Centres locaux de développement (CLD) et des Conférences régionales des élus (CRÉ), peuvent non seulement affecter la capacité d'action et de planification des intervenants locaux en termes de ressources disponibles, mais influencer considérablement la capacité de mobilisation et de résilience des communautés.

\section{MISE EN CONTEXTE DES MESURES ACTUELLES}

Les transformations récentes dont il est question ici ont connu une accélération soudaine en raison des décisions politiques des derniers mois. Aussi est-il fondamental de préciser que ces trans- formations s'inscrivent dans un processus dit de «redéfinition du rôle étatique », plus large et dilué dans le temps. Un phénomène qu'on retrouve depuis de nombreuses années dans 
plusieurs pays, en Europe, comme en Amérique du Nord (Esping-Andersen, 2002 ${ }^{3}$ ).

Comme le rappelle Benoit Lévesque : «[...] la question de la "fonction de base et du nouveau rôle des pouvoirs publics" est toujours d'actualité. Le néolibéralisme a, sans doute, réalisé une fonction historique bien précise : celle de remettre en question le paradigme de l'État qui avait cours jusqu'alors, mais il n'a pas réussi à fournir un nouveau paradigme dont la légitimité se serait imposée largement, voire quasi naturellement [...] » (Lévesque, 2003, p. 490).

Dans une perspective plus large, les transformations en cours ne sont certainement pas seulement porteuses de problèmes et de difficultés. La restructuration du rôle de l'État et l'émergence d'autres acteurs, déjà présents et encastrés dans la société, entrainent également des effets positifs et des occasions d'innovation sociale favorables. «[...] De nouveaux acteurs sociaux, voire une nouvelle gauche, proposent un renouvèlement de l'État et de la société civile pour répondre aux nouveaux problèmes, aux nouvelles demandes sociales et aux nouvelles valeurs. Ce faisant, les positions, maintenant en présence, ne se limitent pas à "plus d'État" versus "plus de marché". En conséquence, on peut même avancer l'hypothèse d'un nouveau paradigme en (lente) émergence qui permettrait de repenser les rapports entre l'État, le marché et la société civile» (Lévesque, 2003, p. 490). Certains auteurs reconnaissent un «[...] participation- nisme insufflé par la reconnaissance du rôle de la société civile dans la gouverne de certaines instances de la société. Ce nouvel esprit [...] favorise le déploiement d'un ensemble d'innovations qui contribuent à la définition et à l'actualisation du modèle néolibéral de régulation $»^{5}$ (Fontan, 2011, p. 26). Il y a donc un espace relativement ouvert à des circonstances opportunes et à des formes d'innovation sociale, au moins potentiellement capables de répondre à des besoins - anciens ou nouveaux - provenant des territoires : soit des quartiers urbains, soit des régions éloignées. La dimension sociale de ces innovations concerne différents types d'actions - individuelles, collectives, organisationnelles et institutionnelles - qui se mettent en place quand un nouvel usage social est construit (Fontan, 2011).

\section{La restructuration de l'État et l'émergence d'autres acteurs, déjà présents et encastrés dans la société entrainent également des effets positifs et des occasions d'innovation sociale favorables.}

Une question que nous poserons donc dans cet article a trait aux conditions par lesquelles les innovations sociales peuvent, au moyen de la participation et de la mobilisation, se produire et se perpétuer dans un contexte caractérisé, en premier lieu, par une redéfinition du rôle étatique et, en second lieu, par une volonté de rationalisation des dépenses publiques.

\section{UN ESSAI ${ }^{6}$ DE DÉFINITION DE L'INNOVATION SOCIALE}

\section{Les cinq formes d'innovation : de produit, de procédés, de modes de production, de débouchés et de matières premières, peuvent par certains aspects contenir des formes d'innovation sociale.}

L'innovation sociale est un concept qui, dans la tradition universitaire et intellectuelle, est plutôt resté dans l'ombre. Ce terme n'est cependant pas complètement nouveau. Le concept plus général d'innovation, qui a été beaucoup plus utilisé, et pas seulement en économie, possède en fait des dimensions et une portée sociale importantes.
Dans sa large conception de l'innovation, Schumpeter inclut des éléments fortement liés au social. Les cinq formes d'innovation : de produit, de procédés, de modes de production, de débouchés et de matières premières, peuvent par certains aspects contenir des formes d'innovation sociale. À cet égard, comme le rappellent Polanyi (1949) et Granovetter (1985), il y a, entre l'économique et le social, une relation étroite, prenant la forme d'un encastrement/désencastrement ${ }^{7}$ Dans cette perspective, nous pouvons alors comprendre que toute forme d'innovation (économique, technologique, etc.) présente des dimensions sociales et dépend de l'adaptation et 
de la nouvelle configuration des institutions sociales. En ce qui concerne plus spécifiquement la sociologie, le concept d'innovation est présent de manière plus indirecte et surtout dans l'œuvre de Tarde (1890), qui explique l'évolution des sociétés comme un cumul d'innovations quotidiennes, capables d'apporter du changement ${ }^{8}$ (Fontan, 2004, cité dans Leclair, 2013; Howaldt et al., 2015). Le social devient ici la dimension principale de l'innovation. Il n'est plus limité à l'environnement où l'innovation prend forme. Il s'agit d'un processus dynamique où le social organise et structure les réponses aux différents problèmes et difficultés liés à la transformation de la relation entre la redistribution (l'État), l'échange (le marché) et la réciprocité (famille et liens sociaux). Ces réponses, structurées socialement au niveau local, peuvent, même en combinaison avec d'autres initiatives - politiques, mesures d'entreprises, etc. - devenir des solutions viables à des problèmes auxquels l'État et/ou le marché n'arriveraient pas à faire face. Évidemment, toute nouvelle proposition ne peut pas devenir ipso facto une innovation sociale. L'innovation n'est pas forcément la nouveauté ou ce qui découle d'un simple changement. Il faut plutôt la voir comme une augmentation de rationalité sociale et politique, visible pour les acteurs impliqués dans le processus ${ }^{9}$ (Donolo et Fichera, 1988, p. 77). Fontan (2011) identifie trois dimensions centrales qu'une nouvelle proposition devrait comporter pour être désignée comme innovation :

- Une finalité clairement définie comme réponse à une question, à un enjeu et/ou à un problème, afin de proposer un changement positif;

- Une stratégie sociale de mobilisation $^{10}$ à plusieurs niveaux (local, national, institutionnel, citoyen, etc.) pour créer une opinion publique favorable autour de l'initiative/action collective;

- La proposition, la négociation ou l'imposition d'un choix politique concernant les modalités d'usage nouvelles susceptibles de devenir ou non une innovation sociale.

Comme l'explique clairement l'auteur : «l'innovation s'établit premièrement dans la définition d'une finalité puis dans un "habillement socio- politique" de cette proposition par un travail laborieux, complexe et exigeant de socialisation et [de] politisation de l'usage social proposé par l'innovateur ou le groupe innovant $»^{11}$. (Fontan, 2011, p. 29)

Quand on traite de l'innovation sociale, qu'il faut toujours voir comme un processus, il y a plusieurs éléments à prendre en considération. D'abord, le contexte dans lequel l'innovation a lieu, avec ses limites et ses contraintes. Ces obstacles peuvent être d'ordre relationnel - en ce qui concerne par exemple les interactions entre les individus, la cohésion sociale ou au contraire un manque de concertation et de participation de la population; économique - en termes de ressources financières ayant un rôle, par le biais des initiatives et des activités, sur le développement d'une innovation; politique - telle qu'une incompréhension de la portée innovatrice d'une initiative ou une opposition pour des intérêts électoraux ou de lobbying.

Ensuite, il faut considérer les acteurs impliqués dans le processus d'innovation (Fontan, 2011), qu'ils soient les créateurs ou les proposeurs de l'innovation (rôle important du leadership, individuel ou collectif), les participants ou les bénéficiaires.

Puis, en ce qui concerne plus directement la mobilisation, l'empowerment agit sur les relations entre les individus et les groupes dans les territoires, là où elles sont encastrées ${ }^{12}$, et les change en positif (Vicari et Moulaert, 2009, p. 179). C'est donc dans ce sens qu'on peut parler d'innovation territoriale.

Un autre aspect important touche la gouvernance, laquelle est également en relation avec la participation et la mobilisation des communautés. L'innovation sociale recèle une approche pragmatique des problèmes qui suppose plusieurs techniques de gestion et de gouvernance. Parfois, cela se fait par des modèles d'hybridation qui s'inspirent de l'approche managériale. L'innovation sociale implique l'usage de nouvelles formes d'organisation et de gouvernance, où l'approche «bottom-up » coexiste avec d'autres formes de socialité plus globales et quelquefois même virtuelles. Ces réseaux se structurent en fait autour des nouvelles technologies et des 
médias sociaux. De cette manière, les relations sociales deviennent des instruments à mobiliser afin d'obtenir des résultats (Murray et al., 2010).

Enfin, le dernier élément de l'innovation sociale se rapporte évidemment aux résultats. Avant d'en arriver à des résultats définitifs, qui ne sont pas toujours faciles à mesurer en revanche, on en passe par des expériences et des prototypes. L'idée est de mettre à l'épreuve l'innovation sociale, soit dans la pratique, soit à la faveur de tests plus formels (Murray et al, 2010). Une fois validée, l'innovation sociale commence à devenir un usage commun, qu'il faut socialiser à travers des stratégies de diffusion et de mobilisation. Quelquefois, une activité de « lobbying » devient également nécessaire pour convaincre l'acteur public - ou, dans certains cas le marché - de reconnaitre l'innovation. Si l'innovation est efficace et largement socialisée, elle devrait à long terme être en mesure d'amener un changement du système de référence (mouvements sociaux, modèle socioéconomique, lois, valeurs, attitudes, comportements, etc.). Cet objectif représente le but ultime d'une innovation sociale (Murray et al., 2010).

\section{UN EXEMPLE DE MOBILISATION EN TEMPS D’AUSTÉRITÉ : LES INITIATIVES DE SOUTIEN AUX PROCHES AIDANTS AU BAS-SAINT-LAURENT}

La santé est un domaine dans lequel on peut facilement trouver des exemples de reconfiguration et d'adaptation qui concernent, comme mentionné plus haut dans les sections précédentes, la sphère de la redistribution (rôle de l'État), de l'échange (rôle du marché et du secteur privé dans la santé) et de la réciprocité (rôle des familles et des communautés - associations, OSBL, économie sociale, etc.). Dans le cas du Québec, par exemple, depuis une vingtaine d'années on parle de virage ambulatoire. Un pas important dans cette direction a été accompli surtout avec la Politique de soutien à domicile «Chez soi : le premier choix » introduite par le MSSS au Québec, en $2003^{13}$ (Ducharme, 2006). Dans ce système, le milieu de vie devient le lieu privilégié de dispensation des services sociaux et de santé aux personnes âgées. Nous sommes ici en présence d'un clair changement de paradigme des fonctions providentielles et de l'engagement étatique $^{14}$ (Leseman, 2002; Ducharme, 2006) par rapport aux autres acteurs concernés : en premier lieu la famille - encore souvent pensée comme un ensemble de responsabilités déclinées au féminin et la communauté. Notre but ici n'est pas de critiquer ce modèle, mais simplement de rappeler que l'offre de services est encore souvent très peu adaptée aux besoins des familles ${ }^{15}$ (Dumarche, 2006, Lero et al., 2012). Par exemple, en ce qui concerne le soutien psychosocial pour l'aidant.e, mais aussi, plus simplement, un répit en soirée, les fins de semaine ou quelques heures pendant la journée, afin de faciliter la conciliation travailfamille-soin et vie personnelle.

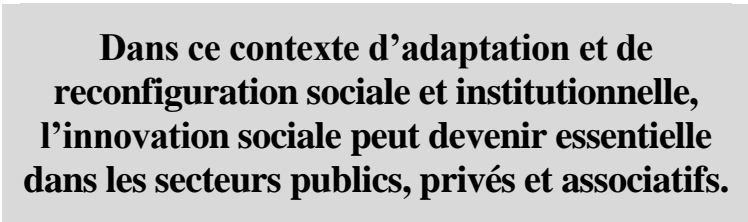

Dans la majorité des cas, les services semblent encore cibler presque exclusivement l'ainé. Le contexte (familial) du soin est généralement négligé par les politiques publiques. Bien que dans les discours on énonce souvent la nécessité de répondre aux besoins des familles, des mesures concrètes tardent à être mises en œuvre (Dumarche, 2006). Les outils présents sont souvent conçus comme une offre de dernier recours, ce qui contribue au développement, du côté des familles, d'une certaine réticence à utiliser le peu de services offerts (Dumarche, 2006; Alberio et Tremblay, 2016 ${ }^{16}$ ).

Dans ce contexte d'adaptation et de reconfiguration sociale et institutionnelle, l'innovation sociale peut devenir essentielle dans les secteurs publics, privés et associatifs. Par exemple, depuis quelques années (autour de 2010) au Bas-SaintLaurent, comme également en d'autres régions du Québec, on observe une importante mobilisation autour de cette question émergente des proches aidants. Tout d'abord à travers une prise de conscience dans plusieurs organismes publics et communautaires (centres-femmes, entreprises d'économie sociale, CLSC, CSSS), où les intervenants ont identifié, de manière indivi- 
duelle, au cours des années, l'émergence de ce besoin :

«C'est des situations qu'elles vivaient soit avec leur père, leur mère, leur conjoint, et qu'elles trouvaient ça lourd. Donc on s'est dit qu'il fallait faire quelque chose avec elles, ces dames-là. Moi et mes collègues on était sensibles à cette question. On est trois sur quatre à être proches aidantes : je suis proche aidante, la coordonnatrice est proche aidante, et l'autre travailleuse est aussi proche aidante. Moi j'ai fait de l'intervention et tout ça, sauf qu'il n'y avait pas d'activité spécifique en tant que telle pour les proches aidants. Fait qu'à un moment donné, on a entendu parler pendant quelques années que L'Appui Bas-Saint-Laurent offrait des subventions pour aider spécifiquement les proches aidants. À ce moment-là, on a demandé un projet et ça a été accepté ». (Intervenante Centre-femmes)

D'une manière plus concertée, une mobilisation s'est dans quelques cas développée autour des tables des ainés, actives dans plusieurs MRC du Québec, dans le cadre des initiatives MADA (Municipalité amie des ainés) ${ }^{17}$, rassemblant une variété d'acteurs: institutionnels, communautaires et citoyens. Lors de ces tables de concertation, les acteurs ont identifié plusieurs enjeux pour lesquels il fallait trouver des solutions. Le soutien aux proches aidants a donc été identifié comme l'une de ces priorités. Cette démarche a aussi été encouragée par la Conférence régionale des élus (CRÉ), par le biais de l'APPUI, un organisme de soutien aux proches aidants fondé en $2010^{18}$.

«Dans notre MRC, nous avons avancé à partir du diagnostic de la population vieillissante. Dans un modèle initial, il y avait même des signatures d'engagement. Nous, on n'a pas été jusqu'à des signatures d'engagement; c'était déjà une notion d'engagement assez forte. Avec la table sur le vieillissement, on est rendu à 4 enjeux. C'est rendu une grosse table de partenaires. On a dû aller vers un comité de coordination. Ça grossit pas mal, et moi, je ne peux pas faire toute seule, les liens partout. Ensuite, un des quatre enjeux, le comité des proches aidants, a pris son envol aussi suite au fait qu'il y avait des sous pour faire des projets (Projet Appui-CRE). Le travail préliminaire de terrain, avec le diagnostic, avait déjà été fait avant. Donc, les gens voulaient vraiment plus travailler ensemble, à ce niveau-là des proches aidants.

Ça fait qu'on a fait une cartographie de tous les services dans la MRC pour faire encore plus de réseau ». (CSSS et membre du comité proches aidants)

Comme il ressort clairement de cet extrait d'entrevue, l'intérêt et la capacité de mobilisation autour d'une problématique quelconque représentent un premier pas décisif vers des initiatives potentiellement porteuses d'innovation sociale. Cependant, cette capacité de mobilisation ne peut pas être tenue pour acquise. Elle peut varier beaucoup selon les caractéristiques physiques, sociales et économiques des territoires. Dans les régions plus «éloignées » et moins peuplées, par exemple, même pour des raisons logistiques (transport, conditions des routes l'hiver, etc.) mobiliser la population autour d'un enjeu de longue durée n'est pas du tout évident. De plus, dans certains territoires, les ressources humaines, associatives et institutionnelles nécessaires au développement d'actions collectives n'existent pas toujours.

En ce qui a trait plus particulièrement aux ressources financières, même si parfois, dans une première phase, la mobilisation et les actions se font de manière plutôt spontanée, sans que les acteurs prennent forcément en considération la disponibilité de fonds et de financements; or celle-ci devient au fil du temps une condition fondamentale. Pour que l'initiative innovante puisse se formaliser, s'affirmer et continuer, il est en fait nécessaire d'avoir un cadre bien précis d'actions et, évidemment, de ressources financières :

«Ce qu'on faisait pour aider ces personnes-làn'était pas ciblé, ce n'était pas encadré dans un plan d'action ou dans un projet, mais sauf qu'on pouvait quand même apporter du soutien, il y avait quand même de l'écoute, il y avait quand même des groupes de soutien, des initiatives pour 
l'aide à domicile, etc. Chez nous on offre une formation qui s'appelle Antidote. Cela fait longtemps que ça existe dans les Centres-Femmes. C'est une approche féministe qui fait qu'on organise des groupes sur l'estime de soi, sur l'affirmation, la confiance en soi. C'est dans ces groupes de soutien là que je pouvais identifier qu'il y avait des proches aidantes, mais ce n'était pas ciblé "proches aidants". Après on a soumis un autre projet, qui était au ministère de la Justice, ça s'appelait "Mobilisation des collectivités" et touchait un peu plus de proches, mais pas encore directement la question des proches aidants. Le tout s'est fait très progressivement $»$. (Intervenante Centre-Femmes)

Pour revenir au sujet de la mobilisation, qui est pour nous à la base même d'une possibilité d'innovation sociale, il ne faut pas oublier que les ressources économiques ne servent pas seulement à développer des projets concrets, mais également à soutenir et à maintenir la participation autour des enjeux. Selon la grande majorité des acteurs que nous avons rencontrés, cette mission risque de devenir encore plus difficile dans le contexte actuel, caractérisé par une incertitude des ressources financières, mais aussi par une méconnaissance de la volonté gouvernementale. En fait, les acteurs du milieu ne semblent pas pleinement comprendre la vision et les objectifs derrière les mesures d'austérité, ou les réformes visant l'équilibre budgétaire, comme préfère les appeler le gouvernement.

«Dans le fond, le soutien financier aux organismes communautaires n'a pas évolué depuis des années. Là, il est encore plus à risque avec la réforme en cours. Alors que c'était toujours des enveloppes qui arrivaient par des voies et avec des montants différents chaque année, ce qui entraine des problèmes de planification. Aujourd'hui, on entend beaucoup le terme

\section{CONCLUSION}

L'innovation sociale n'est pas quelque chose d'évident à contrôler et à reproduire d'un territoire à l'autre. Elle est en fait encastrée dans un contexte socioterritorial, économique et "pérennité". Ça, je suis plus capable. "Pérenniser", comme si, du moment qu'on retire une enveloppe budgétaire, on était capable de pérenniser une action sans budget. En plus, les questions financières deviennent périlleuses en termes de concertation dans les milieux locaux. Il y a rien de pire que de mettre un peu d'argent autour d'une table pour tuer une concertation. Ça fait qu'il faut faire attention, il faut être très délicat. Surtout quand ces ressources sont insuffisantes. Pour moi, en tout cas, le partenariat, la mobilisation des acteurs, est essentiel, s'il n'y en a pas, je ne travaille plus. Il y a plus rien qui va se passer. » (CSSS et membre du comité proches aidants)

Dans un contexte d'austérité, les mesures peuvent prendre plusieurs formes. Il ne s'agit pas toujours de coupes directes ou de fermetures d'organismes, comme dans le cas des Centres locaux de développement (CLD) ou des Conférences régionales des élus (CRE). La « rationalisation » des dépenses passe aussi par des contraintes d'évaluation. Bien évidemment, il est essentiel de lier le financement à des formes de contrôle des actions et de leur efficacité. Cependant, comme le rappelle cet intervenant, des critères «objectifs » et très standardisés ne sont pas toujours faciles à identifier et risquent surtout de mal s'appliquer à la participation et à la mobilisation autour d'enjeux tels que l'empowerment des individus et des communautés : «Le bailleur de fonds, il veut justifier les fonds, et c'est normal, je peux comprendre ça. Mais la partie pour laquelle la communauté $s$ 'inscrirait vraiment dans un changement ne rentre pas toujours dans une évaluation. Elle est souvent très difficile à mesurer, même si les effets dans la communauté sont très visibles». (Intervenant communautaire, membre du comité proches aidants)

politique spécifique. Chaque territoire, pas seulement à l'échelle régionale, mais aussi au niveau de chaque MRC ou village, présente des ressources humaines et communautaires propres, 
qu'on ne peut pas toujours retrouver ailleurs; même dans un village proche. Il s'agit par exemple d'un leadership individuel ou collectif qui arrive pour plusieurs raisons -institutionnelles, sociales ou économiques - à s'organiser et à mobiliser la communauté sur un territoire.

Dans cette perspective, nous estimons que les mesures d'austérité risquent de diminuer les occasions favorables de participation et de mobilisation citoyennes : des conditions nécessaires au développement de l'innovation sociale. Ces changements peuvent aussi creuser les inégalités à l'intérieur des territoires (entre les populations) et parmi les territoires mêmes.

Des structures telles que les Conférences régionales des élus (CRE) ou les Centres locaux de développement (CLD) aujourd'hui en voie de disparition ou de réorganisation, selon les MRC, ont favorisé à travers les années la mise en place d'une gouvernance régionale permettant aux communautés locales, aux organismes et aux associations de structurer progressivement des initiatives dans plusieurs secteurs, y compris les services sociaux; comme notre exemple sur les proches aidants le démontre.

Ces démarches s'inscrivent dans la tradition du développement local québécois, basé entre autres sur la reconnaissance des acteurs communautaires. Le rôle de ces acteurs a été historiquement considéré essentiel pour un vaste territoire comme le Québec, formé par quelques grands centres et par une majorité de régions, définies éloignées.

Comme nous l'avons rappelé dans cet article, les changements actuels s'insèrent dans un long processus. Depuis plusieurs années, on observe un mouvement contradictoire, qu'on pourrait presque définir comme un paradoxe. D'un côté, l'État redéfinit son rôle en limitant de plus en plus son investissement financier dans la société. De l'autre, inspiré par un fort interventionnisme, le politique ne renonce pas à des formes de contrôle. Nous pouvons en retrouver des exemples dans les changements que la CRÉ a vécus dans les dernières décennies. En 2004, on passe des Conseils régionaux de développement (CRD) aux Conférences régionales des élus (CRÉ). Ce premier changement effectué par le gouvernement libéral de l'époque ne concerne pas simplement la dénomination. Il transforme tout d'abord l'équilibre des pouvoirs, en renforçant le palier politique au détriment de la représentation et de la participation de la société civile. Un pas ultérieur dans cette direction se traduit par les réformes en cours : «L'objectif de cette nouvelle enveloppe destinée aux MRC est de leur donner des leviers supplémentaires pour intervenir en matière de développement rural, économique et régional, et d'aménagement dans un esprit de souplesse et d'imputabilité. Il appartiendra aux MRC de prendre les décisions en ces matières de façon autonome, au bénéfice des citoyens qu'elles représentent $»^{19}$. En utilisant un vocabulaire qui renvoie à la rationalité et à la flexibilité, le gouvernement semble vouloir augmenter le contrôle politique, dans un contexte caractérisé par la réduction budgétaire. Cela risque de faire de cette cession de compétence une sorte de cadeau empoisonné pour les élus locaux.

Pour conclure, ces réformes nous semblent fragiliser des espaces importants de dialogue et de participation entre les institutions - au sens large du terme - et les citoyens. De plus, l'acteur public semble aussi refuser un rôle de facilitateur et de promoteur des processus d'innovation sociale déjà «encastrés » dans la société, en préférant plutôt le rôle de contrôleur des ressources, en attribuant notamment aux MRC des enveloppes dont on ignore encore la portée.

\section{Ces réformes nous semblent fragiliser des espaces importants de dialogue et de participation entre les institutions - au sens large du terme - et les citoyens.}

Pour terminer sur une note - semi - positive, les milieux ont souvent - mais malheureusement pas toujours - une bonne capacité de résilience qui fait en sorte que la mobilisation et les effets positifs de l'innovation sociale peuvent durer un certain temps. Cependant, cette capacité ne peut pas se pérenniser longtemps sans l'apport de ressources humaines et financières requises. 
« Le contexte actuel, il est difficile. Mais, en même temps, ça me rassure, parce que je vois que les solidarités, même dans le contexte actuel, ça demeure. D'après moi, c'est ce qui va nous permettre de passer au travers de ce qu'on est en train de vivre dans toutes ces réformes... Mais la base était là avant. Par contre, le problème est que cela n'est pas le cas pour tous les milieux. Nous, si on n'avait pas travaillé de cette façon-là, je pense qu'on ne passerait pas à travers ce qui s'en vient. » (CSSS et membre du comité proches aidants)

\section{BIBLIOGRAPHIE ET NOTES}

'Équilibre budgétaire, tarifs des services de garde, abolition des Centres locaux de développement (CLD), abolition/réorganisation des Conférences régionales des élus, etc.

${ }^{2}$ Il s'agit d'entretiens effectués entre l'automne 2014 et l'hiver 2015 auprès d'intervenants et d'acteurs locaux, dans le cadre d'une recherche plus large portant sur la question des travailleurs proches aidants dans la région du Bas-Saint-Laurent. Une comparaison avec la région de Montréal (en collaboration avec Diane-Gabrielle Tremblay) est aussi prévue.

${ }^{3}$ Esping-Andersen, G. (2002). Why we need a new welfare state? Oxford, Oxford University Press, 276 pages.

${ }^{4}$ Lévesque, B. (2003). « Fonction de base et nouveau rôle des pouvoirs publics : vers un nouveau paradigme de l'État ». Annals of Publics and Cooperative Economics, vol. 74, p. 489-514.

${ }^{5}$ Fontan, J.M. (2011). «Développement territorial et innovation sociale», Dans Innovation sociale et territoriale. Convergences théoriques et pratiques. Sous la dir. de Guy Bellemarre, Juan-Louis Klein. Québec, PUQ, p. 26.

6"Social innovation is a term that almost everybody likes, but nobody is quite sure of what it means" (Pol/Ville 2009, cité dans Howaldt et al., 2015).

${ }^{7}$ Pour un approfondissement : Hédoin, C. (2008). «Le concept d'encastrement : un essai d'application aux marchés financiers ", https://rationalitelimitee.wordpress.com/2008/09/2 0/le-concept-dencastrement-un-essai-dapplicationaux-marches-financiers/

${ }^{8}$ Leclair, L.A. (2013). «L'entrepreneuriat social et l'innovation sociale comme facteurs fédérateurs du système national d'innovation ». Dans Boutiller, S., Djellal, F., Uzunidis, D. L'innovation : analyser, anticiper, agir. Éd. P.I.E. Peter Lang.

Howaldt, J. et Schwarz, M. (2015). Social Innovation: Concepts, research fields and international trends.
Forschung und Beratung in betrieblichen und regionalen Innovationsprozessen. Münster: LIT Verlag.

${ }^{9}$ Donolo, C., Fichera, F. (1988). Le vie dell'innovazione: Forme e limiti della razionalità politica, Milano, Feltrinelli, p. 77.

${ }^{10}$ Cet élément sera central dans notre analyse.

${ }^{11}$ Fontan, J.M. (2011), p. 29.

${ }^{12}$ Vicari Haddock, S., Moulaert, F. (2009). Rigenerare la città : Pratiche di innovazione sociale nelle città europee, (A cura di), Il Mulino, p. 179.

${ }^{13}$ Ducharme, F. (2006). Famille et soins aux personnes âgées : Enjeux, défis et stratégies, Québec, Beauchemin. ${ }^{14}$ Leseman, F. (2002). "Vieillissement de la maind'œuvre, dynamiques d'entreprises et politiques : évolutions d'un itinéraire de recherche ». Dans Ruptures, segmentations et mutations du marché du travail. Sous la dir. De Tremblay, D.G., Dagenais, L.F., Sainte-Foy, PUQ.

${ }^{15}$ Lero, D., Spinks, N., Fast J., Tremblay, D-G. (2012). «The availability and effectiveness of workplace supports for Canadian caregivers », Edmonton, University of Alberta, Research on Aging, Policies and Practice (RAPP), Available on, http://www.rapp. ualberta.ca/ en/ /media/rapp/Home/Documents/Proj_ Workplace.pdf

${ }^{16}$ Les travailleurs proches aidants. Le cas de la région du Bas-Saint-Laurent et de Montréal.

${ }^{17} \mathrm{https} / / /$ www.mfa.gouv.qc.ca/fr/aines/mada/pages/inde x.aspx

${ }_{18}$ $\mathrm{Au}$ Bas-Saint-Laurent il s'agit d'une table de concertation régionale intersectorielle qui se donne comme mandat la concertation et la mobilisation des personnes et des organisations concernées par le soutien aux personnes proches aidantes de personnes aînées en perte d'autonomie. La région du Bas-Saint-Laurent reçoit un soutien financier annuel de la Société de gestion du fonds de soutien aux proches aidants créé en 2009 par le gouvernement du Québec et la Famille Lucie et André Chagnon. Cette enveloppe dédiée est gérée de façon à soutenir les communautés locales et la région dans la consolidation et le développement de services spécifiquement dédiés aux personnes proches aidantes de personnes aînées ». http://www.lappui.org/bas-saintlaurent/pour-les-organisations/a-propos

${ }^{19} \mathrm{http} / / / \mathrm{www}$. mamrot.gouv.qc.ca/developpementregional-et-rural/prochaine-gouvernanceregionale/foire-aux-questions-abolition-des-cre/ 